\title{
Endothelial dysfunction in the early- and late-stage type-2 diabetic Goto-Kakizaki rat aorta
}

Emi Kazuyama, Motoaki Saito, Yukako Kinoshita, Itaru Satoh, Fotios Dimitriadis and Keisuke Satoh

Division of Molecular Pharmacology, Tottori University Faculty of Medicine, 86 Nishimachi, Yonago, 683-8503, Japan

Key Words: diabetes; Goto-Kakizaki (GK) rat; aorta; nitric oxide synthetase; aging Running head: GK diabetic rat aorta

Correspondence:

Motoaki Saito, MD, PhD

Department of Pathophysiological and Therapeutic Science, Division of Molecular Pharmacology, Tottori University Faculty of Medicine, 86 Nishimachi, Yonago, 683-8503, Japan

Telephone: +81-859-38-6162

FAX: $\quad+81-859-38-6160$

e-mail address: saitomo@med.tottori-u.ac.jp 


\begin{abstract}
As there are increasing evidences that human diabetes induces cardiovascular dysfunction, we investigated the type 2 diabetes-induced endothelial dysfunction in the early and late-stage Goto-Kakizaki (GK) rat aorta. We performed organ bath studies, and examined the changes in expression levels of muscarinic $\mathrm{M}_{3}$ receptor, endothelial, inducible and neuronal nitric oxide synthetase (eNOS, iNOS and nNOS, respectively) mRNAs in the rat aorta utilizing real-time polymerase chain reaction in 12-weeks-old and 70-week-old GK rats as well as in age-matched Wistar rats. In the 12-week-old GK rat aorta, a significant increase in norepinephrine-induced contraction and a significant decrease in acetylcholine-induced relaxation as well as significant increases in expression levels of muscarinic $\mathrm{M}_{3}$ receptor and eNOS and a significant decease in nNOS mRNAs were observed compared to age-matched controls. In the older GK rat aorta, significant decreases in acetylcholine- and nitroglycerine-induced relaxations as well as significant decreases in the expression levels of muscarinic $\mathrm{M}_{3}$ receptor, eNOS, iNOS and nNOS mRNAs were observed compared to those in the younger GK rats. In contrast, although significant decreases in acetylcholine and nitroglycerine-induced relaxations were observed, the expression levels of muscarinic $\mathrm{M}_{3}$ receptor, eNOS, iNOS and nNOS mRNAs in the older Wistar rats aorta were unchanged, increased, increased and decreased, respectively, compared to the younger Wistar rat aorta. These results indicate that endothelial dysfunction in the rat aorta progresses with age and development of diabetes condition, and that decreased relaxations in the late-stage rat aorta may be due to these alterations.
\end{abstract}




\section{Introduction}

It has been estimated that there will be 200-300 million cases of metabolic syndrome including type 2 diabetes in Western society by 2010. Moreover, all characteristics of the metabolic syndrome are known to be significant risk factors for cardiovascular diseases [1]. Although several studies have examined vascular dysfunction in patients with diabetes or in animal models of diabetes, there are only limited data describing vascular dysfunction with age of type 2 diabetes animals [2]. Endothelial cells in the aorta play an important roll in the control of vascular smooth muscle and auto-regulation of vascular homeostasis through the release of a variety of vasconstricting and vasodilating autacoids, including thromboxan, prostacyclin, nitric oxide (NO) [3,4]. In 1980, Furchgott and Zwadaki reported that the relaxation of isolated rabbit aorta and other arteries induced by acetylcholine (ACh) and other agonists for muscarinic receptors depended on the presence of endothelial cells in the preparation [5]. In the diabetic rat aorta, endothelium-dependent vasodilatation is impaired, largely due to the production of vasoconstrictor prostanoids and/or oxygen-derived free radicals [6]. It is also thought that hyperglycemia, oxidation of low-density lipoprotein, hypertension, hypertriglyceridemia and autonomic neuropathy promote vascular dysfunction in diabetes patients [6].

It has been reported that NO derived from endothelial cells is an important mediator of vasodilatation [7]. Abnormal release of, or response to, NO has been proposed to contribute to vascular and endothelial dysfunction in the diabetic state [8]. Furthermore, it has been reported that the muscarinic $\mathrm{M}_{3}$ receptor mainly mediates vasodilatation via the actions of eNOS in the rat aorta $[9,10]$.

The mildly diabetic Goto-Kakizaki (GK) rat, which is obtained by selective breeding 
of individuals with glucose intolerance from a non-diabetic Wistar rat colony, is a widely accepted, genetically determined rodent model for human type 2 diabetes [11]. Impaired response to glucose both in vivo and in isolated islets of the young GK rat [12] and raised plasma levels of glucagons in GK rats are common features of human type 2 diabetes and contribute to its development [13]. GK rats are characterized by early mild insulin resistance, followed by abnormalities in insulin secretion and modest hyperglycemia [14], and this model has been shown to be useful for studying type 2 diabetic complications [15].

Because information concerning age-related endothelium dysfunction in diabetic type 2 animal models is limited, we conducted a longitudinal examination using this model. In order to clarify the long-term influence of diabetes type 2-induced vascular dysfunction and reveal its molecular mechanisms, we investigated aortic relaxation and contraction as well as the aortic expression levels of the mRNAs for muscarinic $\mathrm{M}_{3}$ receptor and NO synthase (NOS) of three isotypes (eNOS, iNOS, nNOS) in the earlyand late-stage GK rat. 


\section{Materials and methods}

\section{Animals}

All animal experiments were performed in accordance with the guidelines established by the Tottori University Committee for Animal Experimentation. Six-week-old male GK and Wistar rats were purchased from SLC (SLC, Shizuoka, Japan). All groups were kept under identical conditions, with free access to food and drinking water. The rats were divided randomly into 4 groups (n=6-8 each): groups A and B consisted of 12-week-old Wistar and GK rats respectively, and groups C and D consisted of 70-week-old Wistar and GK rats, respectively. Upon reaching 12 or 70 weeks of age, the rats were sacrificed with an overdose of pentobarbital (60 mg/animal, i.p.). Blood samples were collected from the vena cava. The aorta was removed from each animal and immediately used for functional studies or frozen at $-80^{\circ} \mathrm{C}$ until used to measure the expression levels of muscarinic $\mathrm{M}_{3}$ receptor and NOS mRNAs.

\section{Serum glucose and insulin measurement}

Serum glucose concentrations in the experimental rats were measured by the hexokinase method using a Glucose CII kit (Wako Pure Chemical, Osaka, Japan) according to the manufacturer's instructions. Insulin concentrations were measured by ELISA using a Rat Insulin ELISA kit according to the manufacturer's instructions (Mercodia AB, Uppsala, Sweden).

\section{In vitro organ bath experiments}

The functional studies were performed according to our previous report [16]. The thoracic aortas were cut into approximately 3-mm-long ring segments. Each ring 
was suspended on a wire hook in an organ bath $(25 \mathrm{ml})$ containing Krebs-Henseleit solution, and bubbled with $5 \% \mathrm{CO}_{2}$ and $95 \% \mathrm{O}_{2}\left(37^{\circ} \mathrm{C}\right)$. One hook was suspended from a transducer (Mitutoyo, Tokyo, Japan). Each ring was equilibrated unstretched for 30 min. A load of $0.5 \mathrm{~g}$ was applied to each ring by micrometer adjustment, and the load was readjusted to this level 30 min later. Changes in the tone were recorded by a force transducer on a personal computer (Macintosh G3; Apple Computer, Cupertino, CA) by use of Chart v 3.6 .9 software and a PowerLab/16sp data acquisition system (AD Instruments, Castle Hill, Australia). Following a 30-min period of equilibration, the rings were exposed to $100 \mathrm{mM} \mathrm{KCl}$. In the aorta rings, the contractile response to norepinephrine $\left(1 \times 10^{-9}-3 \times 10^{-6} \mathrm{M}\right)$ was determined cumulatively. After a 30 -min washout period, propranolol $\left(1 \times 10^{-6} \mathrm{M}\right)$ was added to prevent the involvement of $\beta$-adrenoceptors. Endothelium-mediated relaxation was measured as a concentration-response curve to acetylcholine $\left(1 \times 10^{-8}-3 \times 10^{-5} \mathrm{M}\right)$ in rings contracted with the submaximal dose of norepinephrine $\left(3 \times 10^{-7} \mathrm{M}\right)$. Endothelium-independent aortic relaxation in response to nitroglycerin (NTG) $\left(1 \times 10^{-6} \mathrm{M}\right)$ was also measured in the rings.

\section{Real-time polymerase chain reaction (quantification of muscarinic $\mathbf{M}_{3}$ receptor, eNOS, iNOS and nNOS mRNAs)}

Expressions of muscarinic $\mathrm{M}_{3}$ receptor, eNOS and iNOS and nNOS mRNAs in the experimental aorta were measured by real-time polymerase chain reaction (PCR) method. The mRNAs were purified using an RNeasy Mini Kit (Quiagen, Valencia, CA) according to the manufacturer's instructions. A reverse transcriptase mixture (28 $\mu \mathrm{l})$ containing $2 \mu \mathrm{g}$ of total RNA was made and incubated at $37^{\circ} \mathrm{C}$ for $60 \mathrm{~min}$. Fifteen $\mu \mathrm{l}$ of 
the master mix was used for the real-time PCR, which was carried out using a LightCycler thermal cycler system with a LightCycler-FastStart Hybridization Probe kit according to the manufacturer's instructions (Roche Diagnostics, Tokyo, Japan). The muscarinic $\mathrm{M}_{3}$ receptor (GeneBank Accession: NM_012527) primers and probe sequences were as follows: primer forward (1227-1245: 5'-GGACTGTGGATGT GGAGAG-3'), primer reverse (1358-1375: 5'-CGAGGAGTT GGTGTCAGA-3'); and probe forward (1267-1284: 5'-CCA GAAGAGCATGGGTGATGGTGACAACT-3'), probe reverse (1286-1325: 5'-XGTCAGAAGGATTTCACCAAGC-TTC CCATCCT-3'). The primer and probe of the $\beta$-actin (GeneBank Accession: NM_031144), eNOS (GeneBank Accession: AJ011116), iNOS (GeneBank Accession: D44591) and nNOS (GeneBank Accession: NM_052799) were from a LightCycler-Primer/Probe Set (rat) (Roche Diagnostics, Tokyo, Japan). A total of $5 \mu \mathrm{l}$ of template was used for the sample. The specificity of the reaction was confirmed by $2 \%$ agarose gel electrophoresis. The primers for the $\beta$-actin gene were used as the internal standard and gene levels were analyzed by real-time PCR using the same reverse transcriptase mixture.

\section{Measurement of $\mathrm{NO}_{2}-\mathrm{NO}_{3}$. concentrations in the aortas}

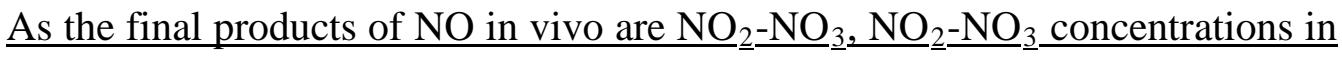
the aortas of 70-week-old GK and Wistar rats were measured by means of the Griess method. In brief, the tissue was homogenized with a Multi-beads Shocker ${ }^{\circledR}$, and then centrifuged at $10,000 \times \mathrm{g}$ for $20 \mathrm{~min}$. The supernatant solution was centrifuged again at

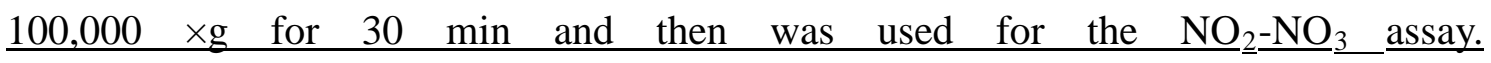
$\underline{\mathrm{NO}}_{2}-\underline{\mathrm{NO}}_{3}$ concentrations were measured by colorimetric assay according to the 
manufacturer's instructions (Nitrate/ Nitrite Colorimetric Assay Kit; Cayman Chemical Co., Ann Arbor, MI). The absorbance was measured at $540 \mathrm{~nm}$. The concentrations were standardized per amount of protein in the tissue. The protein was measured using a commercial kit (Protein Assay Rapid Kit Wako; Wako Pure Chemical, Osaka, Japan).

\section{Data analysis}

The data for the contractions induced by norepinephrine were normalized by the contractions induced by $100 \mathrm{mM} \mathrm{KCl}$. The relaxation responses with submaximal contraction caused by $3 \times 10^{-7} \mathrm{M}$ norepinephrine were expressed as percentages of its contraction. The $\mathrm{EC}_{50}$ and $\mathrm{E}_{\max }$ values were obtained using a Macintosh computer (G3) loaded with Chart v3.6.9 software and a PowerLab/16sp data acquisition system. The $\mathrm{EC}_{50}$ values were calculated as geometric means, whereas the $\mathrm{E}_{\max }$ values were calculated as arithmetic means. The expressions of muscarinic $\mathbf{M}_{3}$ receptor, eNOS, iNOS and nNOS mRNAs were quantified according to the expression of $\beta$-actin mRNA in the experimental rat aorta. A statistical comparison of differences between groups was performed using analysis of variance and Fisher's multiple comparison tests. $\mathrm{P}<0.05$ was regarded as the level of significance.

\section{Drugs and chemicals}

Propranolol and norepinephrine were purchased from Sigma (St. Louis, MO), and NTG (millisrol®) was purchased from Nihonkayaku Co., Ltd. (Tokyo, Japan). All other chemicals were available commercially and of reagent grade. 


\section{Results}

\section{Animals}

The data obtained for body weight and serum concentrations of insulin and glucose in the experimental animals are shown in Table 1 and Figure 1 . The GK rats showed significantly smaller weight gain during the experimental period compared to the Wistar rats. The serum glucose concentrations were significantly elevated in the GK rats compared to the Wistar rats both in the younger and the older animals (Table 1). The serum insulin levels in 12-week-old GK diabetic rats were significantly higher than those in 12-week-old Wistar rats, while the 70-week-old GK diabetic rats had significantly lower serum insulin levels than did the control rats. In 70-week-old Wistar rats, the serum insulin levels were statically significant larger than those in the younger Wistar rats, and in 70-week-old GK rats, the serum insulin levels were statistically smaller than those in younger GK rats.

\section{In vitro organ bath experiments}

The maximum contraction $\left(\mathrm{E}_{\max }\right)$ values and the $\mathrm{EC}_{50}$ values for the contractile responses of the aorta rings to norepinephrine and $\mathrm{KCl}(100 \mathrm{mM})$ were determined. The Emax values in response to norepinephrine in the younger GK rats were significantly higher than those in age-matched Wistar rats, while the $E_{\max }$ values in the older GK rats were similar to those in age-matched Wistar rats (Table 2). The $\mathrm{EC}_{50}$ values in the older GK rats were significantly lower than those in the younger GK rats and age-matched Wistar rats. The maximum relaxation values and the $\mathrm{EC}_{50}$ values in response to ACh are shown in Table 2 . In the aortic rings contracted by norepinephrine $\left(3 \times 10^{-7} \mathrm{M}\right)$, ACh produced relaxation in a dose-dependent manner (Fig. 2). The maximum relaxation 
tension in response to ACh in the 12-week-old GK rats was significantly higher than those in the age-matched Wistar rats. The maximum relaxation tension in response to ACh in the 70-week-old GK rats was significantly higher than those in the age-matched Wistar rats and in the 12-week-old GK rats (Fig.2). The maximum relaxation values in response to ACh in the 70-week-old Wistar rats were significantly lower than those in the 12-week-old Wistar rats (Table 2). The $\mathrm{EC}_{50}$ values in response to $\mathrm{ACh}$ in the older Wistar and GK groups were similar to those in the younger groups (Table 2). NTG-induced endothelium-independent relaxation was decreased in 70-week-old rats compared to the 12-week-old rats both in the GK and Wistar rats, while there were no significant differences in NTG-induced relaxation between the younger GK and Wistar rats (Table 2).

Quantification of muscarinic $M_{3}$ receptor, eNOS, iNOS and nNOS mRNAs in the aorta

The expression levels of muscarinic $\mathrm{M}_{3}$ receptor, eNOS, iNOS and nNOS mRNAs in the GK rat aorta are shown in Figure 3. The expression level of the muscarinic $\mathrm{M}_{3}$ receptor mRNA in the younger GK rats was significantly higher than that in the age-matched Wistar rats. While there was no significant difference of the expressions of muscarinic $\mathrm{M}_{3}$ receptor mRNA between older GK and Wistar rats, the expression level of the muscarinic $\mathrm{M}_{3}$ receptor mRNA in the older GK rats was significantly lower than that in the younger ones. The expression levels of the eNOS, iNOS and nNOS mRNAs in the older GK rat were significantly lower than those in the younger one. The expression level of eNOS mRNAs was significantly higher in the older Wistar rat than that in the younger one. The expression level of iNOS mRNAs in 
the older Wistar rat was significantly higher than that in the younger Wistar rat and that in older GK rat. The expression level of iNOS mRNAs in the older GK rat was significantly lower than that in the younger GK rat. The expression level of nNOS mRNA in the younger Wistar rat was significantly higher than that in younger GK rat. In contrast, the expression level of nNOS mRNA in the older Wistar rat was significantly lower than in the younger one. The expression level of nNOS mRNAs in the older GK rat was significantly lower than that in age-matched Wistar rat.

\section{$\underline{\mathrm{NO}}_{2}=\underline{N O}_{3}$ concentrations in the late-stage GK and Wistar rats}

$\underline{\mathrm{NO}}_{2}-\underline{\mathrm{NO}}_{3} \underline{3}_{2}$ concentrations in aorta samples of 70 -week-old $\mathrm{GK}$ and

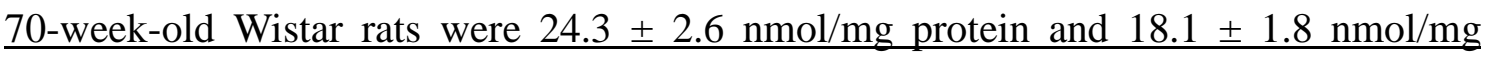
protein, respectively. However, the p value was 0.095, and subsequently the difference between the two groups was statistically non significant. 


\section{Discussion}

The main findings of the present study are that in the late stage (70-week-old) GK rats, endothelium-independent relaxations were impaired compared to those in the younger (12-week-old) GK or age-matched Wistar rats, and that reduced expressions of eNOS, iNOS and nNOS mRNAs were observed in the old GK rats compared to the age-matched Wistar rats, resulting in a decline of relaxation directly via smooth muscle. Furthermore, in this study, we confirmed that 12-week-old GK rats were mild hyperinsulinemic and hyperglycemic, and that 70-week-old GK rats were modest hypoinsulinemic and hyperglycemic. Portha et al. (2009) proposed that the defective beta-cell mass and function in the GK model reflect the complex interactions of multiple pathogenic players: (i) several independent loci containing genes responsible for some diabetic traits (but not decreased beta-cell mass); (ii) gestational metabolic impairment inducing an epigenetic programming of the pancreas (decreased beta-cell neogenesis and/or proliferation) which is transmitted to the next generation; and (iii) loss of beta-cell differentiation due to chronic exposure to hyperglycemia/hyperlipidemia, inflammatory mediators, oxidative stress and to perturbed islet microarchitecture [17].

Vascular dysfunction and cardiovascular diseases are major complications in diabetes mellitus, and are principally caused by endothelial dysfunction [18-20]. Although a number of studies have examined endothelial dysfunction in the metabolic syndrome and/or diabetes, few studies have examined the progression of vascular dysfunction in a long term fashion as these diseases develop [2, 21]. Long-term examination of endothelial dysfunction is necessary, because development of the metabolic syndrome and/or type 2 diabetes occur over months or years as they are 
progressive diseases.

In the present study, aortas from 70-week-old GK rats exhibited decreased endothelium-dependent relaxation in response to ACh in comparison with both age-matched Wistar rats and younger GK rats. These results are in agreement with previous studies conducted on the aorta of Zucker diabetic fatty (ZDF) rats. Oltman et al. (2006) showed that ACh-induced relaxation was attenuated in the aortas of 28- to 40-week-old ZDF diabetic rats in comparison with the aortas of 8- to 12-week-old ZDF rats [2]. Additionally, Zhang et al. (2004) reported decreasing endothelial function over time (3 to 9 months of age) in the ZDF rat aorta [22]. In the present study, although there were no significant differences between 12-week-old GK and age-matched Wistar rats in regard to the maximum relaxations in response to NTG, significant decreases in the maximum relaxations produced by NTG in both 70-week-old GK and 70-week-old Wistar rats compared to the younger groups were observed. This finding might be attributable to the fact that diabetes impairs the endothelium relatively early in its progression, and affects the aortic smooth muscle later. Bitar et al. (2005) reported that there were no significant differences in the maximum relaxation in response to Ach between aortic rings from one-month-old GK rats and age-matched Wistar rats [23]. Both Sandu et al. (2000) and Witte et al. (2002) reported that aortas from rats with long-term GK diabetes show an impaired endothelium-independent relaxation [24, 25]. Our data are consistent with these previous reports. Hyperreactivity to endothelin (ET)-1 is an important issue in diabetic vascular dysfunction. Insulin resistance, an important component of type 2 diabetes, contributes to vascular responsiveness to vasoactive factors. The potent vasoconstrictor ET-1 is chronically up-regulated in diabetes [26]. Elgebaly et al. (2008) reported that vascular insulin resistance occurs in a 


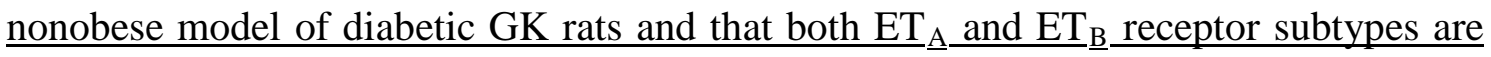
involved in vascular relaxation to insulin [27]. The key factor in endothelial function is NO, which is generated via the constitutive eNOS. There are three distinct isoforms of NOS, and they have been shown to have different localizations and catalytic properties $[28,29]$. The eNOS and nNOS enzymes are suggested to be important regulators of glucagon release [30]. nNOS functionally controls vascular homeostasis [28, 31, 32]. The expression of iNOS is only promoted when endothelial dysfunction occurs [28, 31-33]. eNOS is constitutively expressed and essential for the control of vascular tone [34]. Thus, impaired NO synthesis and/or availability may result in endothelial and vascular wall dysfunction. In this study, we demonstrated that $\mathrm{NO}_{2}=\underline{N O}_{3}$ levels in the aortas of 70-week-old GK (group C) and age-matched Wistar rats (group D) were similar. Bitar et al (2005) suggested a possible mechanism of endothelial dysfunction in the GK diabetic rat. An enhancement in superoxide production and a diminution in NO bioavailability are evident in the GK rat. Diabetes produces a cascade of events involving production of reactive oxygen species from NADPH oxidase leading to oxidation of $\mathrm{BH}_{4} \underline{a n d}_{\text {ancoupling of NOS. This may promote the oxidative inactivation }}$ of NO with subsequent formation of peroxynitrate. An alteration in the balance of these bioactive radicals in concert play a potentially role in pathogenesis of diabetic endothelial dysfunction [23].

In our study, endothelium-dependent relaxation in 12-week-old GK rats was significantly decreased in comparison with the age-matched Wistar rats. In addition, the level of eNOS mRNA expression in 12-week-old GK rats was elevated compared to that in age-matched Wistar rats. Several other investigators have reported similar findings in GK rats. Bitar et al. (2005) demonstrated that ACh-induced relaxation was markedly 
reduced and eNOS protein was significantly increased in GK rats compared to controls [23]. Lee et al. (2009) reported the presence of Akt-independent iNOS expression in the GK diabetic model. They noticed that the defective insulin-induced vasodilation observed in the diabetic vasculature can be restored by the overexpression of active Akt, which advocates a potential novel therapeutic strategy for treating diabetes [35]. Matsumoto et al. (2009) reported that up-regulation of ETA, a defect in ETB-mediated NO signaling, and activation of the MEK/ERK pathway together represent a likely mechanism mediating the hyperreactivity to ET-1 examined in GK diabetic rat mesenteric artery [36].

There have also been reports indicating that eNOS mRNA and protein levels in the aortic wall are increased in diabetic rats [19, 31, 37]. Cai et al. (2005) recently reported that, although total eNOS levels were increased, NO levels were significantly decreased in the aortas of diabetic mice [38]. These data suggest that diabetes may increase eNOS expression and reduce NO bioavailability in the vascular wall [34]. Furthermore, in the present study, we reported for the first time that eNOS mRNA levels were lower in older GK rats than in younger ones. Moreover, our report suggests that the expression levels of iNOS mRNA in 70-week-old Wistar rats were significantly increased compared to those in the younger Wistar rats. In contrast, expression levels of iNOS mRNA in GK rats in the late stage were significantly decreased compared to those in the younger GK rats. It is well known that iNOS is induced by inflammation and/or infiltration of neutrophils and that iNOS-derived NO exhibits a powerful protective activity under a cellular stress condition [38]. In various pathologic conditions, including diabetes mellitus, iNOS is expressed in atherosclerotic plaques and produces a large amount of $\mathrm{NO}$ and $\mathrm{O}_{2}{ }^{-}$locally, which results in production of 
oxidative products [38]. Recently, Ji et al. (2008) reported that increased levels of free radicals with a decrease of vascular endothelial growth factor and iNOS contribute to the progression of the pressure-overload hypertrophied heart to the failing heart [39].

In the 12-week-old GK rats, the expression levels of muscarinic $\mathrm{M}_{3}$ receptor mRNAs were significantly higher than those in the age-matched Wistar rats. We previously reported a similar pattern in the expression levels of muscarinic $\mathrm{M}_{3}$ receptor mRNAs in streptozotocin-induced diabetic rat aortas [16]. This up-regulation of muscarinic $\mathrm{M}_{3}$ receptor mRNAs may be related to a decrease in Ach in the early phase of diabetes. In the later stage of diabetes, the muscarinic $\mathrm{M}_{3}$ receptor mRNA levels in GK rats were returned to similar levels with those of the age-matched Wistar rats with decompensation.

In conclusion, this study has demonstrated diabetes-related and age-related alterations in endothelial function, and expressions of muscarinic $\mathrm{M}_{3}$ receptor, eNOS, iNOS and nNOS mRNAs in the aortas of GK rats and Wistar rats. 


\section{References}

1. Amos AF, McCarty DJ, Zimmet P (1997) The rising global burden of diabetes and its complications: estimates and projections to the year 2010. Diabet Med 14: S1-S85.

2. Oltman CL, Richou LL, Davidson EP, Coppey LJ, Lund DD, Yorek MA (2006) Progression of coronary and mesenteric vascular dysfunction in Zucker obese and Zucker diabetic fatty rats. Am J Physiol Heart Circ Physiol 291: H1780-1787.

3. Furchgott RF, Vanhoutte PM (1989) Endothelium-derived relaxing and contracting factors. FASEB J 3: 2007-2018.

4. Furchgott RF (1999) Endothelium-derived relaxing factor: discovery, early studies, and identification as nitric oxide. Biosci Report 1: 235-251.

5. Furchgott RF, Zawadzki JV (1980) The obligatory role of endothelial cells in the relaxation of arterial smooth muscle by acetylcholine. Nature 288: 373-376.

6. De Vriese AS, Verbeuren TJ, Van de Voorde J, Lameire NH, Vanhoutte PM (2000) Endothelial dysfunction in diabetes. Br J Pharmacol 130: 963-974.

7. Feletou M, Vanhoutte PM (1999) The third pathway: endothelium-dependent hyperpolarization. J Physiol Pharmacol 50: 525-534.

8. Huszka M, Kaplar M, Rejto L, Tornai I, Palatka K, Laszlo P, Udvardy M (1997) The 
association of reduced endothelium derived relaxing factor-NO production with endothelial damage and increased in vivo platelet activation in patients with diabetes mellitus. Thromb Res 86: 173-180.

9. Boulanger CM, Morrison KJ, Vanhoutte PM (1994) Mediation by M3-muscarinic receptors of both endothelium-dependent contraction and relaxation to acetylcholine in the aorta of the spontaneously hypertensive rat. Br J Pharmacol 112: 519-524.

10. Khurana S, Chacon I, Xie G, Yamada M, Wess J, Raufman JP, Kennedy RH (2004) Vasodilatory effects of cholinergic agonists are greatly diminished in aorta from M3R-/- mice. Eur J Pharmacol 493: 127-132.

11. Goto Y, Kakizaki M, Masaki N (1976) Production of spontaneous diabetic rats by repetition of selective breeding. Tohoku J Exp Med 119: 85-90.

12. Salehi A, Carlberg M, Henningson R, Lundquist I (1996) Islet constitutive nitric oxide synthase: biochemical determination and regulatory function. Am J Physiol 270: C1634-1641.

13. Unger RH (1976) The Banting Memorial Lecture 1975. Diabetes and the alpha cell. Diabetes 25: 136-151.

14. Murakawa Y, Zhang W, Pierson CR, Brismar T, Östenson CG, Efendic S, Sima AA (2002) Impaired glucose tolerance and insulinopenia in the GK-rat causes peripheral 
neuropathy. Diabetes Metab Res Rev 18: 473-483.

15. Yagihashi S, Tnosaki A, Yamada K, Kakizaki M, Goto Y (1982) Peripheral neuropathy in selectively-inbred spontaneously diabetic rats: Electrophysiological, morphometrical and freeze-replica studies. Tohoku J Exp Med 138: 39-48.

16. Shinbori C, Saito M, Kinoshita Y, Satoh I, Kono T, Hanada T, Nanba E, Adachi K, Suzuki H, Yamada M, Satoh K (2007) Cyclohexenonic long-chain fatty alcohol has therapeutic effects on diabetes-induced angiopathy in the rat aorta. Eur J Pharmacol 56: 139-144.

17. Portha B, Lacraz G, Kergoat M, Homo-Delarche F, Giroix MH, Bailbé D, Gangnerau MN, Dolz M, Tourrel-Cuzin C, Movassat J (2009) The GK rat beta-cell: a prototype for the diseased human beta-cell in type 2 diabetes? Mol Cell Endocrinol. 297: 73-85.

18. Guerci B, Bohme P, Kearney-Schwartz A, Zannad F, Drouin P (2001) Endothelial dysfunction and type 2 diabetes. Part 2: altered endothelial function and the effects of treatments in type 2 diabetes mellitus. Diabet Metabol 27: 436-447.

19. Hink U, Li H, Mollnau H, Oelze M, Matheis E, Hartmann M, Skatchkov M, Thaiss F, Stahl RA, Warnholtz A, Meinertz T, Griendling K, Harrison DG, Forstermann U, Munzel T (2001) Mechanisms underlying endothelial dysfunction in diabetes mellitus. Circ Res 88, E14-22. 
20. Pannirselvam M, Verma S, Anderson TJ, Triggle CR (2002) Cellular basis of endothelial dysfunction in small mesenteric arteries from spontaneously diabetic (db/db -/-) mice: role of decreased tetrahydrobiopterin bioavailability. $\mathrm{Br} \mathrm{J}$ Pharmacol 136: 255-263.

21. Kobayashi T, Matsumoto T, Ooishi K, Kamata K (2004) Differential expression of alpha2D-adrenoceptor and eNOS in aortas from early and later stages of diabetes in Goto-Kakizaki rats. Am J Physiol Heart Circ Physiol. 287: H135-143.

22. Zhang C, Yang J, Jennings LK (2004) Leukocyte-derived myeloperoxidase amplifies high-glucose-induced endothelial dysfunction through interaction with high-glucose-stimulated, vascular non-leukocyte-derived reactive oxygen species. Diabetes 53: 2950-2959.

23. Bitar MS. Wahid S, Mustafa S, AI-Saleh E, Dhaunsi GS, AI-Mulla F (2005) Nitric oxide dynamics and endothelial dysfunction on type II model of genetic diabetes. Eur J Pharmacol 511: 53-64.

24. Sandu OA, Ragolia L, Begum N (2000) Diabetes in the Goto-Kakizaki rat is accompanied by impaired insulin-mediated myosin-bound phosphatase activation and vascular smooth muscle cell relaxation. Diabetes 49: 2178-2189.

25. Witte K, Jacke K, Stahrenberg R, Arlt G, Reitenbach I, Schilling L, Lemmer B 
(2002) Dysfunction of soluble guanylyl cyclase in aorta and kidney of Goto-Kakizaki rats: influence of age and diabetic state. Nitric oxide 6: 85-95.

26. Chen S, Mukherjee S, Chakraborty C, Chakrabarti, (2003) High glucose-induced, endothelin-dependent fibronectin synthesis is mediated via NF-kappa B and AP-1. Am. J. Physiol. Cell Physiol. 284: C263-C272.

27. Elgebaly MM, Kelly A, Harris AK, Elewa H, Portik-Dobos V, Ketsawatsomkron P, Marrero M, Ergul A (2008) Impaired insulin-mediated vasorelaxation in a nonobese model of type 2 diabetes: role of endothelin-1. Can J Physiol Pharmacol. 2008 86: 358-64.

28. Alderton WK, Cooper CE, Knowles RG (2001) Nitric oxide synthases: structure, function and inhibition. Biochem J 357: 593-615.

29. Calles-Escandon J, Cipolla M (2001) Diabetes and endothelial dysfunction: a clinical perspective. Endocr Rev 22: 36-52.

30. Henningsson R, Alm P, Lindstrom E, Lundquist I (2000) Chronic blockade of NO synthase paradoxically increases islet NO production and modulates islet hormone release. Am J Physiol. Endocri Metabol 279: E95-E107.

31. Papapetropoulos A, Rudic RD, Sessa WC (1999) Molecular control of nitric oxide synthases in the cardiovascular system. Cardiovasc Res 43: 509-520. 
32. Li H, Forstermann U (2000) Nitric oxide in the pathogenesis of vascular disease. J Pathol 190: 244-254.

33. Kibbe M, Billiar T, Tzeng E (1999) Inducible nitric oxide synthase and vascular injury. Cardiovas Res 43: 650-657.

34. Pandolfi A, De Filippis EA (2007) Chronic hyperglycemia and nitric oxide bioavailability play a pivotal role in pro-atherogenic vascular modifications. Genes and nutrition 2, 195-208.

35. Lee JH, Palasia T, Ragolia L (2009) Impaired insulin-mediated vasorelaxation in diabetic Goto-Kakizaki rats is caused by impaired Akt phosphorylation. Am J Physiol Cell Physiol. 296: C327-38.

36. Matsumoto T, Ishida K, Nakayama N, Kobayashi T, Kamata K (2009) Involvement of NO and MEK/ERK pathway in enhancement of endothelin-1-induced mesenteric artery contraction in later-stage type 2 diabetic Goto-Kakizaki rat. Am J Physiol Heart Circ Physiol. 296: H1388-97.

37. Oyadomari S, Gotoh T, Aoyagi K, Araki E, Shichiri M, Mori M (2001) Coinduction of endothelial nitric oxide synthase and arginine recycling enzymes in aorta of diabetic rats. Nitric oxide 5, 252-260. 
38. Cai S, Khoo J. Mussa S, Alp NJ, Channon KM (2005) Endothelial nitric oxide synthase dysfunction in diabetic mice: importance of tetrahydrobiopterin in eNOS dimerisation. Diabetologia 48: 1933-1940.

39. Ji K, Minakawa M, Fukui K, Suzuki Y, Fukuda I (2008) Increased superoxide radical with a decrease in vascular endothelial growth factor and inducible nitric oxide synthase level leads to the progression of left ventricular hypertrophy in a pressure-overload rat heart model. Ann Thorac Cardiovasc Surg 14: 210-217. 


\section{Figure legends}

Fig. 1: Changes of body weight during the experimental period.

Data are shown as the means \pm SEM for 6-8 animals in each group. *Significantly different from age-matched Wistar rats.

Fig. 2: Endothelium-dependent relaxation of rat aortic rings in response to Ach (large panel). Inset: Contractions (means $\pm \mathrm{SEM}$ ) produced by norepinephrine $\left(3 \times 10^{-7} \mathrm{M}\right)$ in each group (small panel). *) significantly different from age-matched Wistar group (group A vs group B, group C vs group D). ${ }^{\dagger}$ ) significantly different from younger group (group A vs group C, group B vs group $\mathrm{D}) .(\mathrm{p}<0.05)$. Data are shown as the means \pm SEM for 6-8 animals in each group.

Fig. 3: The expressions of muscarinic $\mathrm{M}_{3}$ receptor, eNOS, iNOS, and nNOS mRNAs normalized by $\beta$-actin mRNA in the aortas of GK rats

*) significantly different between group $(\mathrm{p}<0.05)$. 
Table.1 General features in the experimental rats.

\begin{tabular}{llll}
\hline & Body weight $(\mathrm{g})$ & Serum glucose $(\mathrm{mg} / \mathrm{dl})$ & Serum insulin $(\mu \mathrm{g} / \mathrm{l})$ \\
\hline A & $350.0 \pm 4.9$ & $153.4 \pm 7.9$ & $1.5 \pm 0.3$ \\
B & $298.3 \pm 5.4^{*}$ & $233.1 \pm 12.3^{*}$ & $2.2 \pm 0.4^{*}$ \\
C & $561.3 \pm 21.7^{\dagger}$ & $132.2 \pm 5.9$ & $2.5 \pm 0.3^{\dagger}$ \\
D & $424.3 \pm 4.2^{* \dagger}$ & $212.1 \pm 8.3^{*}$ & $0.4 \pm 0.1^{* \dagger}$ \\
\hline
\end{tabular}

A: 12-week-old Wistar rats, B: 12-week-old GK rats, C: 70-week-old Wistar rats, D: 70-week-old GK rats. Data are shown \pm SEM of six to eight determinations in each group. *) significantly different from age-matched Wistar group (group A vs group B, group C vs group D). ${ }^{\dagger}$ ) significantly different from younger group (group A vs group C, group B vs group D). $(\mathbf{p}<0.05)$. 
Table 2. Data of contractile and relaxation studies in the rat aorta.

\begin{tabular}{|c|c|c|c|c|c|}
\hline \multicolumn{3}{|c|}{ Contraction } & \multicolumn{3}{|c|}{ Relaxation } \\
\hline \multicolumn{3}{|c|}{ Norepinephrine } & \multicolumn{2}{|l|}{$\mathrm{ACh}$} & \multirow{3}{*}{$\begin{array}{l}\text { NTG } \\
\text { Maximum } \\
\text { relaxation (\%) }\end{array}$} \\
\hline & $\max / \mathrm{KCl}$ & $E C_{50}\left(10^{-8} M\right)$ & Maximum & $E_{50}\left(10^{-8} M\right)$ & \\
\hline \multicolumn{2}{|c|}{$(100 \mathrm{mM})$} & & \multicolumn{2}{|l|}{ relaxation $(\%)$} & \\
\hline $\mathbf{A}$ & $1.56 \pm 0.03$ & $9.06 \pm 2.75$ & $50.34 \pm 2.38$ & $5.85 \pm 0.81$ & $87.05 \pm 1.82$ \\
\hline B & $1.69 \pm 0.03^{*}$ & $8.21 \pm 1.68$ & $39.74 \pm 4.46 *$ & $5.44 \pm 2.21$ & $91.64 \pm 1.19$ \\
\hline $\mathbf{C}$ & $1.55 \pm 0.05$ & $5.36 \pm 0.91$ & $30.92 \pm 2.33^{\dagger}$ & $3.58 \pm 1.04$ & $76.09 \pm 3.72^{\dagger}$ \\
\hline D & $1.66 \pm 0.04$ & $2.30 \pm 0.35^{* \dagger}$ & $22.09 \pm 4.08^{* \dagger}$ & $3.36 \pm 2.52$ & $73.34 \pm 2.77^{\dagger}$ \\
\hline
\end{tabular}

A: 12-week-old Wistar rats, B: 12-week-old GK rats, C: 70-week-old Wistar rats, D: 70-week-old GK rats. Data are shown \pm SEM of six to eight determinations in each group. *) significantly different from age-matched Wistar group (group A vs group B, group C vs group D). ${ }^{\dagger}$ ) $\quad$ significantly different from younger group (group A vs group C, group B vs group D). (p<0.05). Ach: acetylcholine. NTG: nitroglycerin. 
Fig 1

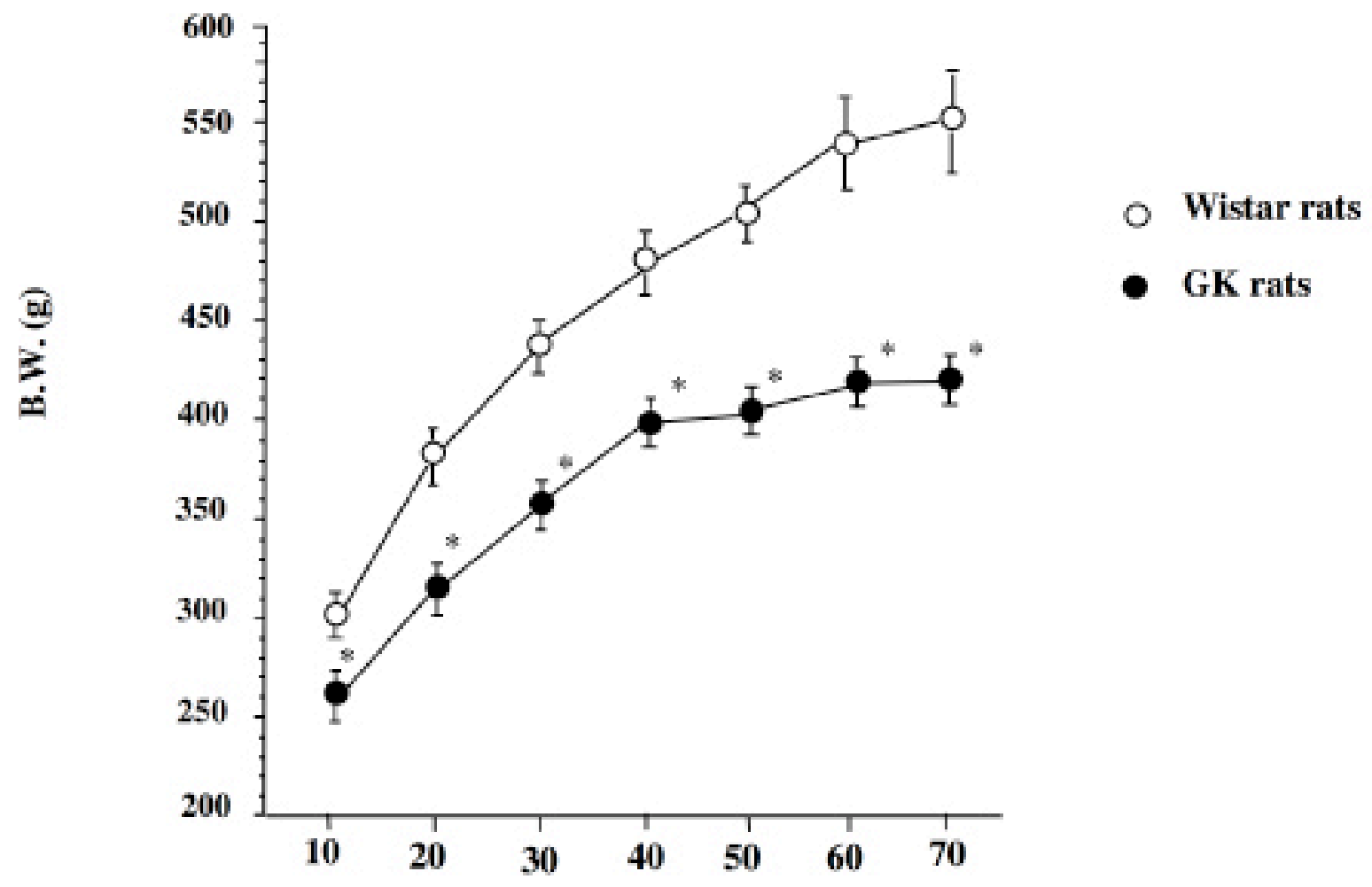

Age (week-old) 
Fig 2

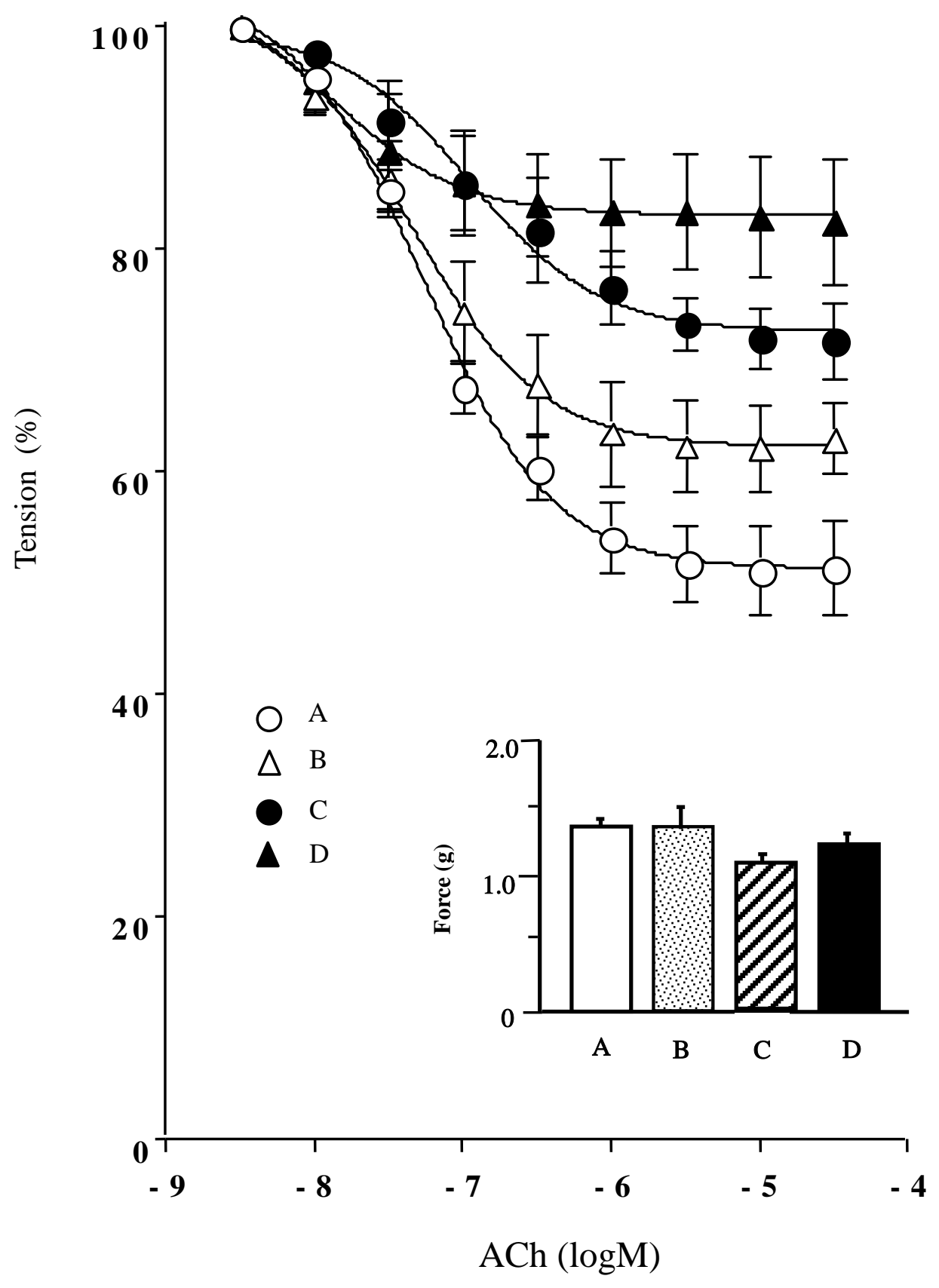


Fig 3
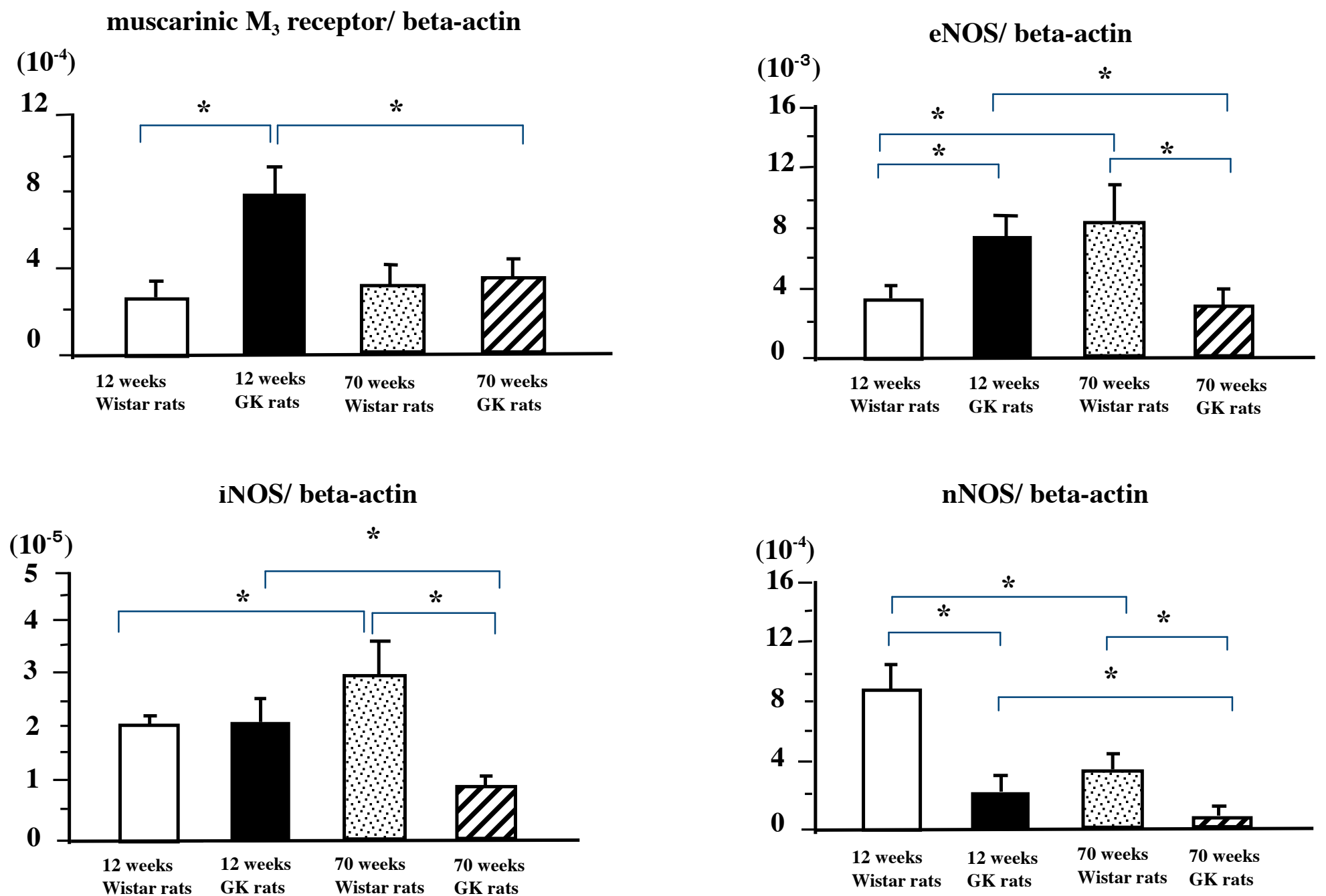